\title{
Biventricular Pacing Improves Clinical Behavior and Reduces Prevalence of Ventricular Arrhythmia in Patients with Heart Failure
}

\author{
Martino Martinelli Filho, Anísio A. A. Pedrosa, Roberto Costa, Silvana A. D. Nishioka, Sérgio F. Siqueira, \\ Wagner T. Tamaki, Eduardo Sosa \\ São Paulo, SP - Brazil
}

Purpose - To analyze the influence of biventricular pacing $(B P)$ on clinical behavior, ventricular arrhythmia (VA) prevalence, and left ventricular ejection fraction ( $L V$ $E F)$ by gated ventriculography.

Methods - Twenty-four patients with left bundle branch block (LBBB) and NYHA class III and IV underwent pacemaker implantation and were randomized either to the conventional or BP group, all receiving BP after 6 months.

Results - Sixteen patients were in NYHA class IV (66.6\%) and 8 were in class III (33.4\%). After 1-yearfollowup, 14 patients were in class II (70\%) and 5 were in class III (25\%). Two sudden cardiac deaths occurred. A significant reduction in QRS length was found with $B P(p=0.006)$. A significant statistical increase, from a mean of $19.13 \pm$ $5.19 \%$ (at baseline) to $25.33 \pm 5.90 \%$ (with BP) was observed in LVEF Premature ventricular contraction prevalence decreased from a mean of $10,670.00 \pm 12,595.39$ SD or to a mean of 3,007.00 $\pm 3,216.63$ SD PVC/24 h with BP $(p<0.05)$. Regarding the hospital admission rate over 1 year, we observed a significant reduction from 60. To 16 admissions with $B P(p<0.05)$.

Conclusion - Patients with $\angle B B B$ and severe heart failure experienced, with BP, a significant NYHA class and LVEF improvement. A reduction in the hospital admission rate and VA prevalence also occurred.

Key words: heart failure, biventricular pacing, ventricular arrhythmias

Heart Institute (InCor), University of São Paulo, Medical School, Brazil Mailing address: Martino Martinelli Filho - InCor- Pacemaker Clinic Av. Dr. Enéas de Carvalho Aguiar, 44 - 05403-000 - São Paulo, SP - Brazil E-mail: martino@incor.usp.br

Received for publication on

Accepted on
Heart failure has become a crucial public health problem because it affects approximately 15 million people worldwide. Moreover, a clear trend toward a cumulative increase in its incidence exists because patients with dilated cardiomyopathy are living longer, a direct consequence of recent promising therapy strategies.

Besides new medical resources, as optional or coadjutant therapy, many nonpharmacological procedures have been introduced lately. Of those is artificial cardiac stimulation that was first described by Hochleitner in 1990 who assessed the role of atrioventricular pacing as a bridge to heart transplantation. Seventeen patients in NYHA Class III or IV were evaluated during short AV delay (100ms). An important clinical and functional improvement was observed.

Further studies showed favorable echocardiographic and hemodynamic findings in patients with heart failure who underwent atrioventricular pacing using variable approaches.

Later, progressing to multisite stimulation, biventricular pacing showed remarkable results with significant improvement in clinical status, quality of life, and functional variables. Despite such documented benefits, the incidence of sudden cardiac death in those patients seems to remain high. The relationship between ventricular arrhythmias and risk of sudden cardiac death has not yet been clearly defined in this population.

The aim of the present study was to assess clinical and functional behaviors as well as the prevalence of ventricular arrhythmias in patients with severe heart failure and biventricular pacing, compared with conventional right atrioventricular stimulation.

\section{Methods}

From January 1997 to October 2000, 24 patients had a biventricular pacemaker system implanted via thoracotomy at our institution to treat severe heart failure. Patients were 
then prospectively randomized to conventional right ventricular or biventricular pacing, and after a 6-month follow-up, crossover was performed.

Patients required the following criteria to be eligible for this study: age > 18 years, irreversible cause of cardiomyopathy, severe heart failure (NYHA III and IV), clinically stable condition for at least 2 weeks, maximal medical therapy for heart failure, left ventricular ejection fraction $<30 \%$ determined with radioisotopic ventriculography (gated blood pool), intraventricular conduction abnormality left bundle branch block (LBBB) registered in at least a 3-lead surface electrocardiogram. A written consent was obtained from all patients, and the protocol was approved by the ethics committee of our institution.

Patients with $2^{\text {nd }}$ and $3^{\text {rd }}$ degree AV block, acute myocarditis, coronary heart disease referred for surgical treatment and unstable angina were excluded from the protocol.

The study consisted of clinical and laboratory evaluations before implantation, and 30,90, and 180 days, and 1 year after the procedure. Heart failure NYHA functional class was assessed at each evaluation. AnECG was undertaken to evaluate QRS length at baseline, and during right ventricular and biventricular pacing. All patients had their AV delay optimized by echocardiography to obtain the best cardiac output. Every patient underwent radionuclide angiography prior to and afterimplantation to assess left ventricular ejection fraction.

Arrhythmia evaluation by 24-hour Holter monitoring was performed prior to and after ( \pm 180 days) biventricular pacing implantation to assess the density and complexity of nonsustained and sustained ventricular tachycardia. All clinical therapies taken before the procedure were maintained, including amiodarone.

Patients were randomized to conventional or biventricular pacing, and crossover was performed 30 days afterwards. Sixty days later, all patients repeated crossover, a new evaluation was made at 180 days, and then patients were followed for 1 year. Rates of hospital admissions during the year before and after the procedure were compared.

All biventricular pacemaker system implantations were performed via thoracotomy due to specific leads for venous access (coronary sinus) being unavailable at the beginning of this study. Two leads were inserted by venous puncture or dissection, respectively, positioned at the right atrium and ventricle. Another lead, via a minimally invasive left thoracotomy, was inserted at the lateral epicardial wall. The atrial lead was connected to the respective pulse generator channel and both ventricular leads to the ventricular channel using a $\mathrm{Y}$ adapter (IS-1 model- Medtronic-IncUSA), the cathode pole being linked to the epicardial lead and the anode pole to the right ventricular lead. One patient had chronic atrial fibrillation and underwent biventricular pacing connecting the atrial channel to the right ventricular lead and the epicardial lead to the ventricular channel of the pulse generator, with the $\mathrm{AV}$ interval programmed to $30 \mathrm{~ms}$.

Data are shown either as mean \pm SD, or median, when appropriate. Nonparametric data were compared with Wilcoxon's test ${ }^{1}$. A p value $<0.05$ was considered statistically significant.

\section{Results}

Seven patients had Chagas' disease (29.2\%), 5 had idiopathic cardiomyopathy (20.8\%), 9 had ischemic cardiomyopathy (37.5\%), 2 had hypertensive heart disease $(8.3 \%)$ and 1 had alcoholic cardiomyopathy (4.2\%). In addition to left bundle- branch block (inclusion criteria), 15 patients (62.5\%) also had $1^{\text {st }}$ degree AV block. Twenty-three patients had sinus rhythm $(95.8 \%)$, and 1 had atrial fibrillation (4.2\%). The study included 23 men and 1 woman. The mean age was 55.08 years, ranging from 37 to 77 years. During enrollment, 16 patients were in NYHA class IV (66,6\%), and 8 were in class III (33.4\%). The follow-up period ranged from 75 to 1489 days $($ mean $=734.2$ ).

Patients' medications consisted of digitalis and diuretics (all), ACE inhibitors (22), carvedilol (7), spironolactone (10), amiodarone (12), and hydralazine and nitrates (3) due to ACE inhibitor intolerance.

The PR interval during basic conditions (sinus rhythm) varied from 140 to $380 \mathrm{~ms}$ ( mean $=225.26 \mathrm{~ms}$ ). QRS length ranged from 124 to $218 \mathrm{~ms}$ ( $m e a n=180.71 \mathrm{~ms}$ ). After conventional RV pacing, $\mathrm{QRS}$ duration increased to a mean value of $217 \mathrm{~ms}$, ranging from 158 to $245 \mathrm{~ms}$, whereas during biventricular pacing, it decreased to a mean of $177.36 \mathrm{~ms}$ (118 to $204 \mathrm{~ms}$ ). When QRS duration was analyzed, a significant difference was found between univentricular and biventricular pacing ( $\mathrm{p}=0.006$ ). The same was not true for the $\mathrm{QRS}$ duration during biventricular pacing compared with the nonpaced QRS $(\mathrm{p}=\mathrm{NS})$.

A significant difference $(\mathrm{p}<0.05)$ occurred in heart failure behavior, considering pre- and postbiventricular pacing conditions. Major improvement was shown in the follow-up period from 90 days to 1 year after implantation. Sixteen patients before stimulation were in NYHA class IV, and 8 were in class III. At 30-day follow-up, 7 patients were in NYHA class IV $(29,1 \%), 8$ were in class III $(33,3 \%)$, and 7 were in class II $(29.1 \%)$. One patient died due to renal failure and another as a consequence of pulmonary infection and sepsis. At 90-day follow-up, 16 patients were in NYHA class II (72.7\%), 3 were in class III (13.6\%), and 1 was in class IV (4.5). Two patients (NYHA class II and IV) experienced sudden cardiac death. After 1-year follow-up, 14 patients were in NYHA class II (70\%), 5 were in class III (25\%), and only 1 patient was in class IV (5\%). The statistical analysis showed a significant NYHA class improvement at the 6-month follow-up visit $(\mathrm{p}<0.05)$.

All patients underwent radionuclide angiography before pacing, with conventional stimulation (right ventricular) and biventricular stimulation. The left ventricular ejection fraction ranged from 13 to 30 (mean $=19.13 \pm 5$.19) before implantation, from 10 to $32($ mean $=19.58 \pm 5.73)$ with right ventricular stimulation, and left ventricular ejection fraction ranged and from 13 to 36 ( mean $=25.33 \pm 5.90$ ) with biventricular stimulation (tab. I). The statistical analysis showed a significant difference between biventricular pacing compared with baseline $(\mathrm{p}<0.05)$.

Ambulatory Holter monitoring was performed in $24 \mathrm{pa}-$ 


\begin{tabular}{|c|c|c|c|c|c|}
\hline \multirow[t]{2}{*}{ Patient } & \multirow[b]{2}{*}{ A } & \multirow{2}{*}{$\begin{array}{c}\text { LVEF } \\
\text { B }\end{array}$} & \multicolumn{3}{|c|}{ HAR } \\
\hline & & & $\mathrm{C}^{*}$ & Preimplant & Postimplant ${ }^{* *}$ \\
\hline 1 & 16 & 17 & 23 & 2 & 0 \\
\hline 2 & 25 & 26 & 31 & 2 & 0 \\
\hline 3 & 18 & 19 & 23 & 3 & 0 \\
\hline 4 & 14 & 14 & 19 & 5 & 2 \\
\hline 5 & 13 & 10 & 17 & 3 & 1 \\
\hline 6 & 25 & 18 & 28 & 3 & 1 \\
\hline 7 & 22 & 24 & 28 & 3 & 1 \\
\hline 8 & 14 & 17 & 22 & 2 & 0 \\
\hline 9 & 22 & 28 & 29 & 3 & 2 \\
\hline 10 & 16 & 16 & 22 & 2 & 0 \\
\hline 11 & 26 & 23 & 32 & 2 & 0 \\
\hline 12 & 17 & 18 & 23 & 2 & 1 \\
\hline 13 & 14 & 12 & 13 & 3 & Death \\
\hline 14 & 22 & 27 & 36 & 2 & 0 \\
\hline 15 & 13 & 14 & 20 & 4 & 2 \\
\hline 16 & 16 & 18 & 22 & 3 & 1 \\
\hline 17 & 17 & 15 & 23 & 3 & 2 \\
\hline 18 & 30 & 32 & 36 & 2 & 1 \\
\hline 19 & 18 & 18 & 28 & 2 & 0 \\
\hline 20 & 16 & 18 & 23 & 2 & 1 \\
\hline 21 & 17 & 18 & 24 & 2 & 0 \\
\hline 22 & 14 & 15 & 22 & 1 & 0 \\
\hline 23 & 26 & 24 & 30 & 2 & 1 \\
\hline 24 & 28 & 29 & 34 & 2 & 0 \\
\hline Total & $19.13 \pm 5.19$ & $19.58 \pm 5.73$ & $25.33 \pm 5.90$ & 60 & 16 \\
\hline
\end{tabular}

tients before pacemaker implantation and 180 days after biventricular pacing. Density and complexity analysis of ventricular arrhythmias was undertaken using sustained ventricular tachycardia or nonsustained ventricular tachycardia records and the number of premature ventricular contractions. Of the 24 patients, $12(50 \%)$ were taking amiodarone (200 to $400 \mathrm{mg}$ ) 6 months before due to nonsustained ventricular tachycardia and also the risk of sudden cardiac death. Twelve patients did not use antiarrhythmic drugs. No patient experienced sustained ventricular tachycardia during follow-up. Mean premature ventricular contraction density was $10,670.24 \pm 12,595.39$ beats, ranging from 78 to 47,954 premature ventricular contractions in 24 hours. There was a mean of $204.56 \pm 613.14$ e.p. nonsustained ventricular tachycardia episodes, ranging from 0 to 3093 before implantation. After biventricular stimulation had started, a significant decrease occurred in the prevalence of premature ventricular contractions to $3,007,69 \pm 3,216.63$ e.p.(mean), ranging from 0 to 10,453 premature ventricular contractions during 24 hours $(\mathrm{p}<0.05)$ (tab. II).

Before pacemaker implantation, the hospital admission rate for heart failure treatment ranged from 1 to 4 per year (mean: $2.52 \pm 0.82$ ) a total of 60 admissions throughout the year before pacemaker implantation. During follow-up with biventricular stimulation, the readmission rate per year decreased to a mean of $0.70 \pm 0.76$, ranging from 0 to 2 per year, showing thus a significant reduction $(\mathrm{p}<0.05)($ tab. I).

\begin{tabular}{|c|c|c|c|c|c|c|}
\hline \multicolumn{7}{|c|}{$\begin{array}{c}\text { Table II - Prevalence of premature ventricular contractions } \\
\text { (before and after biventricular pacing) }\end{array}$} \\
\hline \multirow[t]{2}{*}{ Patient } & \multicolumn{2}{|c|}{$\mathrm{PVC} / 24 \mathrm{~h}$} & \multicolumn{2}{|c|}{ NSVT / $24 \mathrm{~h}$} & \multirow[b]{2}{*}{ Amiodarone } & \multirow{2}{*}{$\begin{array}{l}\text { Dose } \\
(\mathrm{mg})\end{array}$} \\
\hline & A & B & A & $\mathrm{B}$ & & \\
\hline 1 & 12329 & 2624 & 118 & 33 & Yes & 200 \\
\hline 2 & 13742 & 1454 & 59 & 28 & Yes & 300 \\
\hline 3 & 8354 & 3128 & 48 & 18 & No & - \\
\hline 4 & 14567 & 10453 & 34 & 33 & No & \\
\hline 5 & 2164 & 3274 & 24 & 46 & No & - \\
\hline 6 & 78 & 88 & 15 & 18 & No & - \\
\hline 7 & 47954 & 8354 & 3093 & 72 & Yes & 300 \\
\hline 8 & 12995 & 4255 & 68 & 12 & Yes & 200 \\
\hline 9 & 28375 & 7437 & 189 & 39 & Yes & 400 \\
\hline 10 & 436 & 2284 & 10 & 14 & No & - \\
\hline 11 & 3598 & 564 & 21 & 9 & Yes & 200 \\
\hline 12 & 603 & 2 & 2 & 0 & No & - \\
\hline 13 & 35476 & 0 & 114 & 0 & No & - \\
\hline 14 & 7569 & 4032 & 47 & 12 & Yes & 200 \\
\hline 15 & 30020 & 8945 & 156 & 13 & Yes & 300 \\
\hline 16 & 5805 & 317 & 84 & 3 & Yes & 400 \\
\hline 17 & 18328 & 2359 & 146 & 13 & Yes & 300 \\
\hline 18 & 435 & 389 & 2 & 3 & No & - \\
\hline 19 & 5468 & 6532 & 14 & 10 & No & - \\
\hline 20 & 868 & 754 & 20 & 12 & No & - \\
\hline 21 & 10184 & 1681 & 275 & 13 & Yes & 400 \\
\hline 22 & 1410 & 105 & 0 & 2 & No & - \\
\hline 23 & 634 & 132 & 14 & 0 & Yes & 400 \\
\hline 24 & 1907 & 14 & 543 & 5 & No & - \\
\hline
\end{tabular}

\section{Discussion}

Since initial reports on artificial cardiac pacing to treat heart failure, several studies have been performed in an attempt to achieve better results using this alternative therapy. In 1990, Hochleitner ${ }^{2}$ reported 16 patients with endstage dilated cardiomyopathy (mean left ventricular ejection fraction $=16 \%$ ) and NYHA class III or IV, who had cardiac performance improvement with $100 \mathrm{~ms}$ AV delayed pacing programming ${ }^{3}$. However, mortality in those patients did not change. After a follow-up period ranging from 2 to 32 months, 9 sudden cardiac deaths were observed. Despite the high rate of mortality, later confirmed by Brecker et $\mathrm{al}^{4}$, clinical outcomes were encouraging, because NYHA class improvement persisted throughout the follow-up. Recently, biventricular pacing therapy has emerged as a promising adjuvant therapy in cases of interventricular asynchrony marked by left bundle-branch block. Cazeau et al ${ }^{5}$ have shown a 20 to $25 \%$ cardiac output improvement as well as a significant pulmonary wedge pressure decrease related to ventricular resynchronization, seen with radionuclide angiography. With biventricular pacing, a persistent NYHA class improvement was observed after a 3-year follow-up period. Gras et al ${ }^{6}$ studying 69 patients who underwent biventricular pacing graded with the Minnesota Living with Heart Failure Score concluded that quality of life changed from $53 \pm 18$ to $34 \pm 23(\mathrm{p}<0.01)$.

The present study showed persistent NYHA class im- 
provement in 18 patients (75\%) sometimes ameliorating routine daily activities. All patients were receiving optimal medical therapy involving ACE inhibitors, digitalis, and diuretics. Twelve patients (50\%) had been taking amiodarone for at least 6 months before pacing system implantation, which was introduced to treat complex ventricular arrhythmias, a high risk for sudden cardiac death in this subset of patients.

A significant reduction in the prevalence of ventricular arrhythmias $(\mathrm{p}<0.05)$ correlated with an important NYHA class improvement. Otherwise, Hoffmann ${ }^{7}$ and Doval ${ }^{8}$ considered the complex ventricular arrhythmia an independent mortality predictor for sudden cardiac death in patients with severe heart failure.

In our study, the patients had a significant reduction in ventricular arrhythmias with biventricular pacing, mainly in cases of high premature ventricular contraction prevalence and nonsustained ventricular tachycardia episodes $(\mathrm{p}<0.05)$. Besides optimal medical therapy for heart failure, only 12 patients had been taking amiodarone (before and after pacing). These results were clearly related to the cardiac performance improvement and the consequent lower number of hospital admissions. A possible mechanism to justify ventricular arrhythmia reduction with pacing resynchronization could be the dual depolarization wave fronts (right and left simultaneous site). This might decrease ventricular repolarization dispersion and shorten peripheral QRS preventing ventricular reentry, the most important component of the physiopathologic arrhythmia mechanism in patients with cardiomyopathies.

The present study represents an important step in cardiac stimulation evolution regarding heart failure therapy at our institution. It documents our initial experience with biventricular pacing through thoracotomy, which was the only available access at the time of the study design, when reliable venous leads had not yet been developed.

The latest appropriate lead approach has brought for most cases more favorable conditions for biventricular implantation systems. However, nowadays, a defined place for the thoracotomy technique still exists, which must be kept on being used for unsuccessful cases of the venous technique.

Despite the fact that our findings have shown that ventricular resynchronization significantly improves NYHA class, quality of life, and ventricular arrhythmia behavior in patients with severe heart failure and major intraventricular conduction delay, the sudden cardiac death rate is considered high.

To analyze this, further prospective protocols have been recently started, suggesting an association between biventricular pacing and implantable cardioverter defibrillators as an optimal therapy, which may not only improve quality of life but also the longevity of these patients.

\section{References}

1. Rosner B. Fundamentals of Biostatistics. Boston: PWS Publishers, $2^{\text {nd }}$ edition, 1986

2. Hochleitner M, Hortnagl H, Ng CK, et al. Usefulness of physiologic dualchamber pacing in drug-resistant idiopathic dilated cardiomyopathy. Am J Cardiol 1990; 66: 198-202.

3. Hochleitner M, Hortnagl H, Hortnagl H, et al. Log-term efficacy of physiologic dual-chamber pacing in treatment of end-stage idiopathic dilated cardiomyopathy. Am J Cardiol 1992; 70: 1320-5.

4. Brecker SJ, Xiao HB, Sparrow J, et al. Effects of dual chamber-pacing with short atrioventricular delay in dilated cardiomyopathy. Lancet 1992; 340: 1308-11.
5. Cazeau S, Ritter P, Lazarus A, et al. Multisite pacing for end-stage heart failure: Early experience. PACE 1996; 19: 1748-57.

6. Gras D, Mabo P, Tang T, et al. Multisite pacing as a supplemental treatment of congestive heart failure: Preliminary results of the Medtronic Inc. Insync Study. PACE 1998; 21: 2249-55.

7. Hoffmann T, Meinertz T, Kasper W, et al. Mode of death in idiopathic dilated cardiomyopathy: a multivariate analysis of prognostic determinants. Am Heart J 1998; 116: 1455-61.

8. Doval HC, Nul DR, Grancelli HO, et al for the GESICA investigators. Non-sustained ventricular tachycardia in severe heart failure. Independent marker of increased mortality due to sudden death. Circulation 1996; 94: 3198-3203. 